\title{
Pneumococcal serotypes in sputum isolates during acute respiratory illness in Edinburgh
}

\author{
G A GOULD, G B RHIND, A D MORGAN, G Williamson, M A CALDER \\ From the Departments of Respiratory Medicine and Bacteriology, City Hospital, Edinburgh
}

\begin{abstract}
During the years 1978-83 serotyping was carried out on all sputum isolates of pneumococci obtained from patients in the chest wards of the City Hospital, Edinburgh. In 402 patients with acute respiratory illness the peak isolation rates occurred from January to April, and the serotype distribution was similar to that seen in previous UK studies, the commonest types being 3 , $6,9,19,23$, and 8 . The overall mortality rate was $8.7 \%$, the serotype distribution in fatal cases reflecting the distribution of the whole group. The presence of mixed infection, predominantly with Haemophilus influenzae, was associated with a lower mortality rate of $3.5 \%$. Nearly all patients $(92 \%)$ were either elderly or had a chronic underlying disease and only one death occurred in a patient under 70 years who had no pre-existing disease. Of the pneumococcal serotypes isolated from the 292 patients with chronic chest disease, $82 \%$ are included in the new 23 valent pneumococcal vaccine and the efficacy of this needs to be assessed further in high risk patients.
\end{abstract}

\section{Introduction}

The pneumococcus may be present as a commensal of the upper respiratory tract $^{1}$ but is also capable of causing serious infections, particularly in the elderly and in subjects with underlying disease, when it is associated with an appreciable mortality rate. ${ }^{2} \mathrm{~A}$ vaccine containing the purified capsular antigens to 14 distinct pneumococcal serotypes $(1,2,3,4,6 \mathrm{~A}, 7 \mathrm{~F}, 8$, $9 \mathrm{~N}, 12 \mathrm{~F}, 14,18 \mathrm{C}, 19 \mathrm{~F}, 23 \mathrm{~F}$, and 25 ) has been available for some years but, although recommendations for its use have been made, ${ }^{3}$ a unified vaccination policy has not been adopted. In an attempt to improve the efficacy of the vaccine, by providing cover against a wider range of pneumococcal serotypes, a 23 valent vaccine (covering types $1,2,3,4,5,6 \mathrm{~B}, 7 \mathrm{~F}, 8,9 \mathrm{~N}, 9 \mathrm{~V}$, $10 \mathrm{~A}, 11 \mathrm{~A}, 12 \mathrm{~F}, 14,15 \mathrm{~B}, 17 \mathrm{~F}, 18 \mathrm{C}, 19 \mathrm{~F}, 19 \mathrm{~A}, 20,22 \mathrm{~F}$, $23 \mathrm{~F}$, and $33 \mathrm{~F}$ ) has been recently formulated ${ }^{2}$; but the controversy regarding vaccination against pneumococcal disease continues.

We have recorded the pneumococcal serotypes occurring in sputum isolates from patients admitted to our respiratory wards to determine the distribution of serotypes isolated and the associated clinical features, in an attempt to predict the potential value of vaccination in this group of patients.

Address for reprint requests: Dr G A Gould, Rayne Laboratory, City Hospital, Edinburgh EH10 5SB.

\section{Methods}

Using the laboratory records for the years 1978-83 inclusive, we retrospectively studied the case notes of adults admitted to the respiratory wards of the City Hospital, Edinburgh, who had pneumococci isolated from sputum. Our laboratory does not culture or report on salivary specimens, these being identified from the macroscopic appearance and the absence of pus cells and presence of oral squamous on microscopy. In our experience pneumococci are rarely identified in such specimens. Sputum specimens were routinely inoculated on to horse blood agar and heated horse blood agar containing 10 units of bacitracin $/ \mathrm{ml}$ and incubated for 18 hours at $37^{\circ} \mathrm{C}$ in $7 \%$ carbon dioxide. Pneumococci were identified by their typical appearance in the direct Gram film of the specimen, their colonial morphological appearance, their susceptibility to optochin (ethylhydrocuprein hydrochloride), a positive Quellung reaction with pneumococcal Omniserum, and fermentation of inulin. Strains of pneumococci isolated were identified by the Danish typing system, with commercial sera from the Statens Serum Institute, Copenhagen, Denmark. ${ }^{45}$

Cases in which the isolation of pneumococci were considered incidental were not studied further and only those patients with symptoms suggestive of acute lower respiratory tract infection (fever, dyspnoea, cough, sputum production, or pleuritic pain) were 


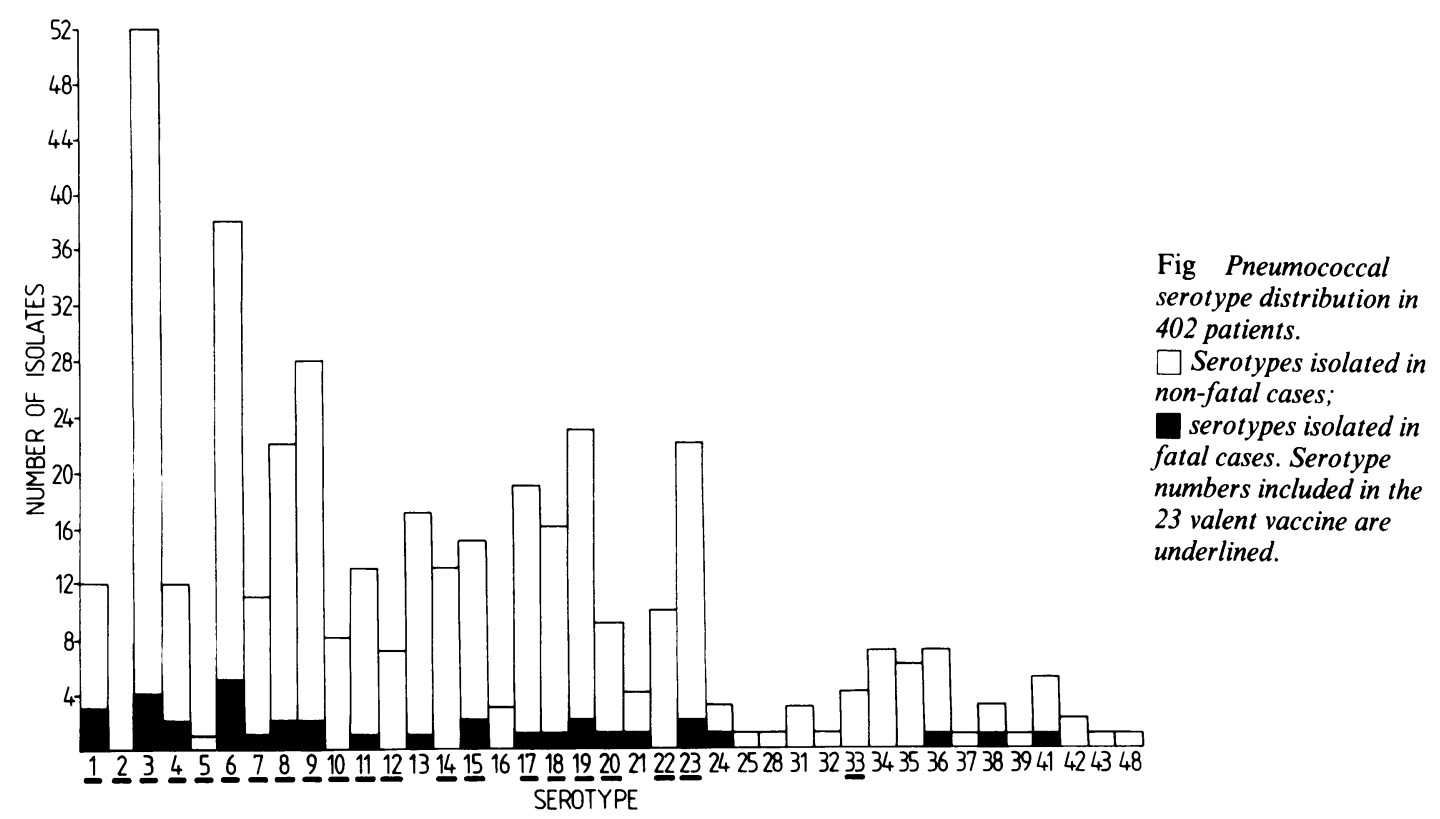

included in the analysis. The following variable were recorded: (1) seasonal variation of isolation rates; (2) distribution of pneumococcal serotypes in different diagnostic groups; (3) frequency of isolation of multiple organisms; (4) clinical outcome, with particular reference to age and other underlying disease processes.

\section{Results}

Pneumococci were isolated from 402 sputum specimens in association with acute respiratory symptoms, the highest isolation rates occurring during the winter months from January to April. Two hundred and ninety two patients had pre-existing chronic chest disease, 54 had other chronic disease (mainly malignancies and cardiovascular and neurological conditions), and 56 had no underlying disease. The mean age of the whole group was 67 years and only 23 patients $(6 \%)$ were under 50 years, 10 of these having underlying chest disease and three others being alcoholic.

Thirty-five deaths (mortality rate $8.7 \%$ ) were attributed either partly or totally to the presence of pneumococcal infection and six deaths were unrelated (four cases of myocardial infarction and two of pulmonary embolism). Only one patient under 70 years with no underlying disease died and only one patient under 50 , a 45 year old male alcoholic. A mixec $\vec{b}$ growth of organisms was obtained in 114 cases, $\mathrm{Hae}^{3}$ mophilus influenzae being found in $102(25 \%)$ of a isolates. Only four deaths occurred in the 114 patients. with multiple organisms (mortality rate $3.5 \%$ ) whereas in the group of patients with a pure growt of pneumococcus the mortality rate was $10 \cdot 8 \%$.

The distribution of serotypes for the whole group ifo shown in the figure, the commonest serotypes being 3 . $6,9,19,23$, and 8 . The serotypes seen in fatal cases largely reflected the overall distribution of serotypes. the presence of mixed infection and the nature of any underlying disease had no obvious effect on serotype distribution.

Serotypes included in the new 23 valent pneu N mococcal vaccine accounted for $83 \%$ of the serotypes isolated from the whole group and $82 \%$ of those isory lated from the 292 patients with chronic chest disease

\section{Discussion}

The patients most at risk of serious pneumococcal infections are generally agreed to be the elderly and those with underlying disease, ${ }^{26-8}$ and recommeri dations have been made for vaccination of certaif high risk groups. ${ }^{2}$ The new 23 valent vaccine was foP mulated on the basis of information from all parts the world and is intended to protect against over $85 \%$ 
of all bacteraemic infections. ${ }^{2910}$ Minor geographical variations in the prevalence of certain serotypes do occur, ${ }^{6-811-16}$ so that production of a single formulation of the vaccine, which is desirable on medical as well as economic grounds, ${ }^{9}$ cannot be expected to cover all infections in all areas. We have therefore studied the pneumococcal serotypes isolated from patients with acute respiratory illness admitted to our chest wards. Most of these patients were suffering from chronic disease, particularly chest disease, and were elderly. The distribution of serotypes in these patients was similar to that seen in other UK studies 681516 ; in addition, the serotypes causing respiratory illness in patients without chest disease were similar to those occurring in patients with chest disease, the precise chest diagnosis being unimportant. The serotypes associated with a fatal outcome reflected the distribution of serotypes in the whole group, suggesting that the main determinant of outcome is the patient and not the organism. Only one death occurred in a patient under 70 years with no underlying disease and only one death in a patient under 50 years, who was an alcoholic.

The mortality rate was lower in the group of patients with mixed infections, most of which were due to $H$ influenzae. This is unlikely to be due solely to a difference in the virulence of the pneumococcus, as the serotypes isolated were the same as those seen in the rest of the group. It might suggest a protective effect of mixed infection, possibly due to population pressure or the antagonistic effects of products of the other organism inhibiting the growth of the pneumococci ${ }^{17}$; but in such a mixed group of patients it is impossible to rule out the effect of other factors, and we can therefore draw no firm conclusion from these mortality rates.

In our patients with chronic chest disease $82 \%$ of the pneumococcal serotypes isolated were included in the new 23 valent vaccine, compared with $60 \%$ in the old 14 valent vaccine. The efficacy of the 14 valent vaccine in certain high risk groups has been demonstrated in several studies, in terms both of its ability to stimulate an antibody response $\mathrm{e}^{\mathbf{1 8 - 2 0}}$ and of protection from pneumococcal infection. ${ }^{21-25}$ Other studies, however, ${ }^{2627}$ and in particular a recent study of 2295 United States veterans, ${ }^{28}$ suggest that the elderly and those with chronic disease may be unable to produce an adequate antibody response to vaccination and consequently derive little or no benefit. Further data on the efficacy of the 23 valent vaccine in such high risk groups is needed before routine vaccination can be recommended.

We wish to thank the consultant physicians at the City Hospital, Edinburgh, for allowing us to publish details of patients under their care.

\section{References}

1 Hendley JO, Sande MA, Stewart PM, Gwaltney HM. Spread of Streptococcus pneumoniae in families. Carriage rates and distribution of types. $J$ Infect Dis 1979;132:55-61.

2 Centers for Disease Control. Update: Pneumococcal polysaccharide vaccine usage-United States. Recommendation of the immunization practices advisory committee. Ann Intern Med 1984;101:348-50.

3 Centers for Disease Control. Pneumococcal polysaccharide vaccine. Recommendation of the immunization practices committee. Ann Intern Med 1982;96:203-5.

4 Austrian $R$. The quellung reaction, a neglected microbiological technique. Mt Sinai J Med 1976;43: 699-709.

5 Menrischen J. The pneumococcal typing system and pneumococcal surveillance. $J$ Infect 1979;1(suppl 2):31-7.

6 Gransden WR, Eykyn SJ, Phillips I. Pneumococcal bacteraemia: 325 episodes diagnosed at St Thomas's Hospital. Br Med J 1985;290:505-8.

7 Ruben FL, Norden CW, Korika Y. Pneumococcal bacteraemia at a medical/surgical hospital for adults between 1975 and 1980. Am J Med 1984;77:1091-4.

8 White RJ, Blainey AD, Harrison KJ, Clarke SKR. Causes of pneumonia presenting to a district general hospital. Thorax 1981;36:566-70.

9 Robbins JB, Austrian R, Lee C-J, et al. Considerations for formulating the second generation pneumococcal capsular polysaccharide vaccine with emphasis on the cross-reactive types within groups. $J$ Infect Dis 1983;148:1136-59.

10 Austrian R. The current status of polyvalent pneumococcal vaccine. Clin Ther 1984;6:572-5.

11 Barry, MA, Craven DE, Finland M. Serotypes of Streptococcus pneumoniae isolated from blood cultures at Boston City Hospital between 1979 and 1982. J Infect Dis 1984;149:449-52.

12 Lamothe F, Delages G, Laverdiere M, Saint-Antoine P. Serogroups and serotypes of pneumococci in Montreal: correlations with age, outcome and indications for vaccination. Can Med Ass J 1984;130:737-40.

13 Bergner-Rabinowitz S, Simchen E, Michel J. Serotype distribution of Streptococcus pneumoniae in hospitalized patients in Israel. Eur J Clin Microbiol 1983; 2:480-2.

14 Hansman D. Serotypes in pneumococcal disease. A ten year study of Australia 1970 through 1979. Aust NZ J Med 1983;13:359-64.

15 MacFarlane JT, Finch RJ, Ward MJ, MacRae AD. Hospital study of adult community-acquired pneumonia. Lancet 1982;ii:255-8.

16 Morgan AD, Rhind GB, Connaughton JJ, Calder MA. Pneumococcal serotyping and antigen detection in pneumococcal pneumonia of adults. J Infect 1984; 9:134-8.

17 Barrett-Connor E. The non value of sputum culture in the diagnosis of pneumococcal pneumonia. Am Rev Respir Dis 1971;103:845-8.

18 Davis AL, Aranda C, Christianson L, Schiffman G. 
Pneumococcal antibodies in patients with chronic obstructive pulmonary disease and their response to pneumococcal capsular polysaccharides: preliminary results [abstract]. Rev Infect Dis 1981;3(suppl):S183.

19 Landesman SH, Smith PM, Schiffman G. Pneumococcal vaccine in elderly patients with COPD. Chest 1983; 84:433-5.

20 Davis AL, Aranda C, Christianson L, et al. Effects of 14-valent pneumococcal polysaccharide vaccine in patients with COPD. Chest 1984;85(suppl):582-3.

21 Shapiro ED, Clemens JD. A controlled evaluation of the protective efficacy of pneumococcal vaccine for patients at high risk of serious pneumonia infections. Ann Intern Med 1984;101:325-30.

22 Broome CV, Facklam RR, Fraser DW. Pneumococcal disease after pneumococcal vaccination: an alternative method to estimate the efficacy of pneumococcal vaccine. N Engl J Med 1980;303:549-52.

23 Austrian R, Douglas RM, Schiffman G, et al. Prevention of pneumococcal pneumonia by vaccination. Trans
Assoc Am Physicians 1976;89:184-94.

24 Riley ID, Tarr PI, Andrews M, et al. Immunization with! a polyvalent pneumococcal vaccine: reduction of adulf respiratory mortality in a New Guinea Highland community. Lancet 1977; :1338-1341.

25 Bolan G, Broome CV, Facklam RR, et al. Pneumococc vaccine efficacy in selected populations in the United States. Ann Intern Med 1986;104:1-6.

26 Austrian R. Surveillance of pneumococcal infection fợ field trials of polyvalent pneumococcal vaccines. Bethesda, Maryland: National Institutes of Healthw 1980. (Report DAB-VDP-12-84.)

27 Williams JH, Moser MM. Pneumococcal vaccine and patients with chronic lung disease. Ann Intern Mejt 1986;104:106-9.

28 Simberkoff MS, Cross AP, Al-Ibrahim M, et al. Efficacir of pneumococcal vaccine in high risk patients. Results of a Veterans Administration cooperative study. $\$$ Engl J Med 1986;315:1318-27. 